\title{
Embryonic-like stem cells derived from postpartum placenta delivered after spotaneous labor emerging as universal prophylactic cancer vaccine
}

\author{
Manole Corocleanu \\ Private, Brasov, Romania; corocleanu@yahoo.com \\ Received 19 October 2013; revised 25 October 2013; accepted 30 October 2013
}

Copyright (C) 2013 Manole Corocleanu. This is an open access article distributed under the Creative Commons Attribution License, which permits unrestricted use, distribution, and reproduction in any medium, provided the original work is properly cited.

\begin{abstract}
In the eighth decade of the last century extensive clinical delayed-type hypersensitivity (DTH) skin tests to an intradermal injection of a pharmaceutical allogeneic human Placenta Suspension (phPS) performed in obstetrical, gynecological and control group patients have shown positive reaction in 239 patients with clinical conditions having been as histopatrhological substratum, a hypoxia-induced adaptive/reactive epithelial cell proliferation, e.g. syncytiotrophoblastic cell hyperplasia, endometrial cell hyperplasia, or different gynecological cancers. Because the immune response against phPS has shown antigenc similarities between normal placental and endometrial hyperplastic cells and different kinds of cancer cells and because many cancers adopt an embryonic stem-like gene expression pattern, it is suggested that the profile of hypoxia-promoting placental and endometrial stem cell proliferation is more embryonic-like, and that the immune respose against phPS is expected to cross-react with tumor cells in vivo. In the process of persistent growth and accelerated oxygen consumption by hyperplastic cytotrophoblastic cells and neoplastic cells in a hypoxic microenvironment, a basic shift in energy metabolism is accompanied by appearance of heat shock proteins (HSPs), of fetal isoenzymes and of membrane glycoproteins (reappearance of oncofetal antigens, OFAs), which, as result of their overexpression/amplification may induce a host immunological response. Thus, it is assumed that phPS prepared from full-term human placentas delivered after a spontaneous labor comprises stem/progenitor cells reverted to a proliferative embryonic stem cell-like-state upon exposure to
\end{abstract}

labor-inducing intrmittent placental hypoxia and that by expressing HSPIOFAs could immunize to generate immune response againjst a variety of antigens that are shared by different kinds of epithelial cancers. This immunological feature of phPS qualifies is as a vaccine-related product that may be used for a preventive cancer vaccine when mixed with a potent adjuvant (BCGVaccine) and given normal healthy individuals.

Keywords: Placenta; Embryonic-Like Stem Cells; Preventive Cancer Vaccine

\section{INTRODUCTION}

The field of tumor immunology has made great advancements in recent years. A retrospective analysis of our previous studies [1] and our proposed pharmaceutical allogeneic human Placenta-Lysates/BCG-vaccine strategy design for cancer prevention [2], based on the assumption that placenta shares identical growth mechanisms, antigenic determinants, and immune-escape properties with cancer cells, combined with present knowledges may provide additional insight in this vaccination approach.

Proteins that are expressed by both malignant and healthy fetal tissues (the placental-fetal complex) are recognized as oncofetal antigens (OFA). These antigens are associated with cell proliferation and differentiation and are produced in high concentrations in pregnancy and malignancy [3]. Their biological role in malignancy is the suppression of the host's immune system, while in pregnancy they affect the maternal immune response, generating maternal tolerance toward the embryo [4]. On the other hand, the involvement of oncofetal antigens in both embryonic and malignant development supports the concept that oncofetal antigens may intervene in the control of maternal immune responses during pregnancy, in 
the manner of the host defense to carcinogenesis [5].

\subsection{Human Embryonic Genes Are Re-Expressed in Cancer Cells and Are Immunogenic}

Marilyn Monk et al. [6] have shown that processes occurring during tumorigenesis may be similar to processes occurring in early development. Human preimplantation embryonic cells are similar in phenotype to cancer cells. Both types of cell undergo deprogramming to a proliferative stem cell state and become potentially immortal and invasive. The exact nature of the initiating event is unknown but, in both processes, development and tumorigenesis, the result is genome-wide demethylation. Therefore, it might be expected that cancer cells will express genes in common with these very early embryonic cells, especially genes specifically associated with deprogramming and return to the undifferentiated and proliferative stem cell state, and the maintenance of that state.

Recently several laboratories have demonstrated that introduction of just four active genes into a mature differentiated cell can convert it into a cell with embryonic stem cell characteristics [7].

Coggin Jr., J. H., et al. [8,9] has identified the OFA/ iLRP protein and its role as a T-cell inducing and immuno-regulating factor in fetogenesis and oncogenesis. This protein was also detected in early to mid gestation fetal cells and emerging trophoblast, hence the term "Oncofetal Antigen". It is concluded that OFA/immature LRP, the $37 \mathrm{kDa}$ fetal-restricted molecule, unlike mLRP (mature LRP, MW $67 \mathrm{kDa}$ ), is a true "immunogenic." In breast cancer patients, Rohrer et al. established several OFA/iLRP-specific CD4+ and cytotoxic as well as regulatory $\mathrm{CD} 8+\mathrm{T}$ cell clones. This phenomenon caused by an excess of the $\mathrm{T}$ cell immunogenic, 37 - $44 \mathrm{kDaOFA}$, enables the immune system to suppress Tc-mediated immunity. In other words, it is an immuno-regulatory controlled measure that prevents overproduction of Tc cells to any Tc-antigen. This immuno-regulation prevents antiself Tc-mediated immunity and other anti-self immunity. The IL-10 inhibited Tc activity and so these cells can dampen anti-tumor immunity of whatever specificity.

During tumor development in mice and humans, oncofetal 32 - $44 \mathrm{kDa} \mathrm{Ag} / \mathrm{immature} \mathrm{laminin} \mathrm{receptor} \mathrm{(OFA/}$ iLRP)-specific Th1, CTL, and IL-10-secreting T-suppressor cells (Ts) cells are induced. The presence of too many Ts or too few effector $\mathrm{T}$ cells appears to predict a poor prognosis $[10,11]$.

\subsection{The Human Discarded Placentas of Healthy Newborns Comprise Embryonic-Like Stem Cells}

Researchers at the University of Pittsburgh (2005) have revealed findings that some placental cells of afterbirth placenta have many of the same characteristics of embryonic stem cells and thus, placenta may yield alternative to embryonic stem cells.

Myoung Woo Lee et al. [12] have shown that in addition to hematopoietic stem cells (HSCs), other potential stem cells (SCs), such as mesenchymal SCs (MSCs), unrestricted somatic SCs (USSCs), cord blood-derived embryonic-like SCs (CBEs), and umbilical cord blood (UCB)derived multipotent progenitor cells (MPCs) have been isolated from Umbilical Cord Blood and characterized.

The newly discovered human cells, named "cord blood-derived embryonic-like stem cells" or CBEs, but also, multipotent non-hematopoietic stem cells are not quite as primitive as embryonic stem cells, which can give rise to any tissue type of the body, but they appear to be much more versatile than "adult stem cells", in that they express some but not all embryonic stem cell markers.

\subsection{The Human Endometrium Comprises Endometrial Regenerative Cells (ERCs)}

Zhong Z. et al. [13] have shown that Endometrial Regenerative Cells (ERCs) are a population of mesenchymal-like cells which possesse pluripotent differentiation capacity and are characterized by unique surface markers and growth factor production. They have some degree of pluripotency similar with bone marrow derived mesenchymal stem cells (MSC) positive for some proteins distinctive of embryonic stem cells. Also, these endometrial derived stem cells possess various characteristics similar to MSC including ability to immune modulate, to induce Treg production and to induce neoangiogenesis. Immunochemical assays of cultured menstrual blood reveal that endometrial cells express embryonic-like stem cell phenotype markers (Oct4, SSEA, Nanong).

\subsection{Hypoxia, Hypoxia-Inducible Factor (HIF) and the Placenta}

Adelman D. M. et al. [14] have shown that placental development is profoundly influenced by oxygen $\left(\mathrm{O}_{2}\right)$ tension. Human cytotrophoblasts proliferate in vitro under low $\mathrm{O}_{2}$ conditions but differentiate at higher $\mathrm{O}_{2}$ levels, mimicking the developmental transition they undergo as they invade the placental bed to establish the maternal-fetal circulation in vivo. Hypoxia-inducible factor-1 (HIF-1), consisting of HIF-1 $\alpha$ and ARNT subunits, activates many genes involved in the cellular and organismal response to $\mathrm{O}_{2}$ deprivation [15].

Placental development is initiated and regulated by the hypoxic uterine environment that is already a hypoxic, $3 \%-5 \% \mathrm{O}_{2}$ versus normal physiologic oxygen tension (normoxia) of approximately $8 \% . \mathrm{O}_{2}$ and the added stress of the limited $\mathrm{O}_{2}$ diffusion stimulates trophoblasts to 
proliferate, migrate, differentiate, and promote maternal and fetal vascular contribution to the placenta $[16,17]$. In the first trimester, establishment of blood flow into the intervillous space is associated with a burst of oxidative stress at a time when placental vascular development is occurring [18].

HIF-1 is a heterodimeric transcript factor that consist of two subunits. The HIF-1 beta subunit is constitutively expressed whereas HIF-1 alfa subunit is regulated by oxigen levels. It is stable under hypoxic conditions but is rapidily degraded under normoxic conditions. After stabilization or activation, HIF-1 translocates to the nucleus where it induces the transcription of numerous downstream genes via their hypoxia response elements. One of the target genes is vascular endothelial growth factor (VEGF).

Recent reports [19] suggest that preeclampsia (PE) is associated with a Th1 predominant profile and may be considered as a failure of the tolerance system allowing the second physiological trophoblastic invasion. This Th1 predominant immunity is closely related to inflamemation, endothelial dysfunction and poor placentation $[10,20]$.

A deficiency of regulatory $T$ cells may play a role in the pathophysiology of preeclampsia. Immunological data [21] have shown that the increased levels of T CD4 (+) 45RO (+) and T CD8 (+) CD25 (+) cells can suggest the activation of CD4 $(+)$ and CD8 $(+)$ T lymphocytes in pre-eclampsia. It seems possible that the activation of $\mathrm{T}$ lymphocytes is associated with the deficiency of $\mathrm{T}$ regulatory cells in PE. A decreased number of T (reg) cells were present in pre-eclampsia, and these changes might break the maternal tolerance to the fetus.

Tereza Cindrova-Davies et al. [22] have shown that labor is a powerful inducer of placental oxidative stress, inflammatory cytokines, angiogenic regulators, and heat shock proteins as result of acute hypoxia/reoxygenation process. These findings are consistent with intermittent perfusion being the initiating cause. Uterine contractions during labor are known to be associated with intermittent utero-placental perfusion. The oxidative stress is a potent inducer of placental synthesis and release of pro-inflammatory factors. At cellular level the transcripts changed in the same direction as observed in preeclampsia, suggesting that the placenta responds similarly to the oxidative stress induced during labor and in preeclampsia.

\subsection{Heat Shock Proteins (HSP) and Immune Function}

HSPs are evolutionarily ancient and highly conserved intracellular molecular chaperones. They are constitutively expressed in the cells, but are highly induced by different stresses such as heat, oxidative stress, oxygen radicals, or transformation. HSPs also perform immune functions. They have the inherent property of binding non-covalently to the peptides generated within cells as a result of degradation of cellular proteins, self and alien. HSP-peptide complexes are intracellular under normal conditions and have a protective function. On the other hand, extracellular located or membrane-bound HSPs mediate immunological functions [23]. It is becoming evident that oxidative stress is at the heart of the regulation mechanisms that maintain a balance between efficient regeneration and proper control of stem cell function. This activity of stem cells, however, has to be carefully controlled, as too much stem cell activity can cause diseases like cancer. Cellular stress may give rise to misfolded or mutated "self" proteins, which in turn result in the presentation of sequences being hidden from the immune system under normal healthy conditions. Such hidden cryptic epitopes of "self" proteins are considered as endogenous danger signal and also "nonself" or new peptides by the host's immune system because of the fact $\mathrm{T}$ cells were not exposed to these hidden epitopes during positive and negative selection in the thymus. A large variety of chaperoned antigenic peptides could be tumor antigens. Such HSPs are expected to carry a repertoire of tumor antigens, some of which would be subdominant or cryptic epitopes and seen by the host's immune system as nonself peptides. These consequences point to a key role of heat shock proteins in fundamental immunological phenomena such as activation of antigen presenting cells, indirect presentation (or cross-priming), and chaperoning of peptides during antigen presentation [24]. Yosino I. et al. [25] in their research conclude that HPS70-reactive CD4+ T cells exist in tumor tissue. These tumor-infiltrating lymphocytes (TIL) recgnize stressed cells and seem to play a Th1-like role that may support antitumor $\mathrm{T}$ cell responses at local tumor sites.

\subsection{The Role of CD40/CD40L in Regulating Immune Response, Epithelial Cell Growth and Differentiation and in Induction of Cell Cycle Blockage and/or Apoptosis of Epithelial Cancer Cells}

Young L. S. et al. [26] have shown that the wide expression of CD40 in normal epithelial cells and carcinoma cells suggests that this receptor has important, additional influences beyond that of regulating immune responses. The major ligand for the external domain of CD40 is CD154 (CD40L), a member of the TNF superfamily. The most abundant source of CD154 is activated $\mathrm{T}$ lymphocytes. While CD40-CD40 ligand interactions are known to regulate $\mathrm{B}$ cell proliferation and differenttiation, much less is known about the role this receptor plays on other cell types, especially those of nonhemopoietic origin. CD40 is expressed and functional on human epidermal basal cells and that, on these cells, CD40 ligation may be a signal for limitation of cell 
growth and induction of differentiation [27]. A direct growth-inhibitory effect can be found when ligated CD40 is on human breast, ovarian, cervical, bladder, non-small cell lung, and squamous epithelial carcinoma cells. This effect is related to the induction of cell cycle blockage and/or apoptosis. The CD40/CD154 couple plays a critical role both in humoral and cellular immune response. The CD40/CD40L system, a key regulator and amplifier of immune reactivity is required for antigen-presenting cell activation as it induces costimulatory molecules and cytokine synthesis. Thus, CD40-CD40L interactions are crucial in the delivery of $\mathrm{T}$ cell help for CTL priming. A natural antagonist of CD40/CD154 interaction is the soluble form of CD40 (sCD40) which has been shown to inhibit the binding of CD154 to CD40 in vitro.. High levels of sCD40 could compete for the ligation of membrane CD40 on CD154 thus resulting in inhibition of antibody production. The rapid up- and downregulation of CD154 on the surface of T cells is an obvious and important way of control. In a first step, CD154 is quickly expressed upon T-cell receptor engagement and in a second step the CD40 itself contributes to downregulating CD154 expression on T cells as sustained interaction between CD40/CD154 leads to endocytosis of the ligand. Although this mechanism is considered to be the major way the CD40/CD154 interaction is down-regulated, the production of a soluble form of CD40 (sCD40) could also be involved, demonstrating a potential antagonistic role for sCD40 in the immune response. However, little is known about the mechanism leading to sCD40 production. The process of shedding is important, as it up-regulates the production of soluble receptors that compete with the membrane receptor for ligand binding, and also reduces the amount of surface receptor, thus modulating the capacity of the cell to signal and thus, inducing immunosuppression. Given the antagonistic activity of sCD40 on the CD40/CD154 interaction, this shedding mechanism might represent an important negative feedback control of CD40 functions [28]. As CD40-CD154 (CD40L) pathway has been shown to attribute to the regulation of T-cell activation, both by independently costimulating $\mathrm{T}$ cells and at least in part by up-regulating CD80/CD86 molecules on APCs, suppression may be generated from fully differentiated Th1 effector cells by stimulation with antigen in the absence of costimulation.

In the light of the above studies, this paper provides further a review and a retrospective analysis in summary of our previous published [1] and unpublished investigations to answer the question whether Placenta Suspension prepared upon Filatov's method from the allogeneic human placenta-tissue after a live full-term delivery, expresses trophoblast cross-reactive antigens present on certain types of trophoblast cells and on transformed cells in the sense that both types of cells express embryonic-like features.

\subsection{Preventive Cancer Vaccine Based on Placental Stem/Progenitor Embryonic-Like Cells of Full-Term Human Placentas Delivered after Spontaneous Labor}

\subsubsection{Background}

Based on the assumption that developing placenta shares identical growth mechanisms, antigenic determinants, and immune-escape properties with cancer cells, immunological cross-reactivity between placental antigens and cancer antigens was investigated.

\subsubsection{Methods Summary}

In the eighth decade of the last century extensive clinical delayed-type hypersensitivity (DTH) skin tests to an intradermal injection in the $1 / 3$ upper anterior surface of the forearm of $0.2 \mathrm{ml}$ of a pharmaceutical allogeneic human Placenta Suspension (ph PS) (Suspensio Placentae pro injectionibus, Odesski zavod Hinfarmapreparatov, Odessa, former USSR), prepared upon Filatov's method from cryopreserved and mechanically disrupted of full term human placentas delvered after spontaneous labor were performed in obstetrical (150 pts.), gynecological (175 pts.) patients with different clinical conditions.

All tests were made under institutional approval and with documented informed consent.

DTH reaction is an immune function assessment that measures the presence of activated T-cells that recognise certain substances. Similar to the mantoux skin test for tuberculosis, a mononuclear cell response is mounted at the site of antigen challenge if the patient has preexisting $\mathrm{T}$ cell immunity.

\subsubsection{Results}

239 patients with different clinical conditions, such as hypertensive disorders during pregnancy (98 pts. with preexisting hypertension, gestational hypertension, preeclampsia, superimposed preeclampsia), abnormal perimenopausal and menopausal uterine bleeding (141 pts.) have shown positive cutaneous delayed-type hypersensitivity (DTH) reactions to phPS.

According to the clinical and histopathlogical diagnosis, two large groups have resulted in which obstetrical and gynecological patients with different clinical conditions have shown positive cutaneous DTH-response to phPS: a group of benign obstetrical and gynecological clinical conditions having as histopathological substratum adaptive syncytiotrophoblast-cell hyperplasia (98 pts.), or reactive/adaptive endometrial cell hyperplasia (76 pts.) and a group of different gynecological cancers (65 pts.). 


\section{CONCLUDING REMARKS}

\subsection{Hypertensive Disorders in Pregnancy Stimulates Proliferation of Cytotrophoblastic Stem Cells That Adopt an Embryonic-Like Stem Cell Antigenic Profile}

Syncytiotrophoblast hyperplasia is commonly seen in patients with hypertension, preeclampsia and occasionally diabetes. Traditionally these clinical conditions have been associated with "placental insufficiency". A compromised maternal circulation in the intervillous space may create a state of true or relative hypoxia that stimulates proliferation of cytotrophoblast stem-like cells that differentiate into the syncytiotrophoblast on the villosities surface in order to increase the exchange area of the placenta. Hypoxic clinical conditions, such as arterial hypertension, diabetes, but also unopposed estrogen stimulation of endometrial growth are also seen in patients with perimenopausal or postmenopausal endometrial cell hyperplasia which have shown positive skin reaction to phPS.

Because the immune response against phPS have shown antigenc similarities between normal placental and endometrial hyperplastic cells and different kind of cancer cells and because many cancers adopt an embryonic stem-like gene expression pattern, it is suggested that the profile of hypoxia-promoting placental and endometrial stem cell proliferation is more embryonic-like. Thus it is assuming that the hyperplastic cells in cytotrophoblastic cell hyperplasia and in abundant endometrial growth, as result of perfusion-limited or diffusion-limited hypoxic clinical conditions, acquire characteristic traits by reactivating genes normally expressed in emerging trophoblast and in transforming cells. It is considered that the potentialities of regeneration of the cytotrophoblast are possible by remodeling the "cytotrophoblast stem-like cells" and that the presence of "adult stem cells" in the endometrial mesenchyme highlights their importance in the regenesis and remodeling of endometrial structures.

\subsection{Labor Intermittent Hypoxia Shifts the Antigenic Profile of Induced Placental Proliferating Stem Cells to One That Is More Embryonic-Like}

We hypothesized also that labor intermittent hypoxiainduced placental stem/progenitor embryonic-like cells of afterbirth placenta could immunize to generate immune response against a variety of antigens that are shared by different kind of epithelial cancers. This raises the exciting possibility of developing a prophylactic vaccine capable of preventing the appearance of various types of cancers in humans.

Hypoxia has shown to play an important role in fa- voring the stem cell state, but also in promoting stem cell proliferation as result of the ability of adult stem cells capable of genomic reprogramming upon exposure to a novel hypoxic environment to adopt the expression profile to one that is more embryonic-like and express some but not all embryonic stem cell markers (multipotent non-hematopoietic stem cells). Also, cancer transformation is intimately coupled with the appearance" of embryonic stem cell-like features, in that both overexpress oncofetal developmental antigens (OFA) and relay exclusively on glucose metabolism for their energy required for rapid cell growth and division.

Persistent growth and accelerated oxygen consumption by hyperplastic placental cells and hyperplastic endometrial cells in a hypoxic microenvironment, a basic shift in energy metabolism is accompanied by appearance of heat shock proteins (HSPs), increase of fetal isoenzymes and of membrane glycoproteins (oncofetal antigens, OFA), which, as result of their overexpression/amplification may induce a host immune response. Both types of cell undergo deprogramming to a embryonic-like stem cell state similar in phenptype to cancer cells. Together, these findings suggest that HIF targets may act as key inducers of a dynamic state of stemness in pathologic conditions and that tissue regeneration after injury appears to recapitulate the pathway of embryonic tissue development.

The up-modulation of OFA gene expression in hyperplastic placental and endometrial cells and in tumors might be related to the requirement of proliferating cells for increased protein synthesis to face the new growth needs of these cells closely related to cell proliferation, that classified it as oncogene and that by overexpression/amplification becomes immunogenic.

The mechanism by which overexpression converts a self proteine into an immunologically recognizable antigen has not been completely elucidated. Most likely the overexpressed protein either becomes more consistently presented to the immune system or the density of presentation crosses the threshold needed for $\mathrm{T}$ cell stimulation.

The abundance of HSPs in the hyperplastic placental and endometrial stem/progenitor embryonic-like cells in response to hypoxia, or in tumor cells may make overexpressed antigens in these proliferative cells intrinsically more cross-presentable to the host DCs for generating protective antitumor activity.

\subsection{Preeclampsia Is Characterizeed by Hypoxia-Reactivated Placental Stem/Progenitor Cells Reverted to a Proliferative Embryonic Stem-Like State and a Prdominant Th1-Cell Immune Profile}

Concerning the preeclampsia, reactivation of the cyto- 
trophoblast as result of placental hypoxia and unusual immune response to self-antigens of the emerging trophoblast, suggests that proinflammatory cytokines and vascular oxidative stress play a role in causing hypertension by activating multiple neurohumoral and endothelial factors and that ligation of CD40 on proliferating extravillous trophoblast stem/progenitor embryonic-like cells, by CD40 ligand (CD154) on CD4+ T cells, may lead to limitation of cell growth and induction of incomplete differentiation and inadequate invasion of the trophoblast and thus, to ineffective vascular remodeling of the uterine spiral arteries that results in insufficient placental perfusion as well as widespread dysfunction of the maternal vascular endothelium [20].

In conclusion, it is assuming that CD4+ T cells frequently recognize nonmutated "self" antigens that are overexpressed by both hyperplastc cytotrophoblastic and endometrial stem cells, but also growing tumor cells. Since patients with positive skin DTH-reaction to phPS can harbor CD4+ $\mathrm{T}$ cells specific for non-mutated, differentiation antigens overexpressed by hyperplastic placental embryonic-like stem cells and neoplastic cells, vaccination might reasonably be expected to amplify the frequency and strength of these pre-exsiting responses or perhaps induce some de novo reactions.

\subsection{Placental Embryonc-Like Stem Cells of Human Full-Term Placentas Delivered after Spontaneous Labor Shares Antigens in Common with Different Kind of Epithelial Cancer Cells and Thus PhPS Emerges as a Preventave Cancer Vaccine-Related Product}

Labor-induced intermittent hypoxia promotes proliferation of placental stem/progenitor cells reverted to an embryonic-like stem cell state by expressing some but not all embryonic stem cell markers and thus, full-term human placenta delivered after spontaneous labor (afterbirth placenta) based on proliferating cord blood-derived embryonic-like stem cells, hypoxia-induced multipotent non-hematopoietic stem cells mixed with other proliferating placental matrix-stem cell populations could immunize to generate immune response againjst a variety of antigens that are shared by different kind of epithelial cancers. The abundance of HSPs in the undifferentiated state of proliferating placental embryonic-like stem cells may make overexpressed OFA antigens in these cells intrinsically more cross-presentable to the host DCs for generating protective antitumor activity both in mother and in newborn. Such a model, although attractive, remains speculative. However, together these findings suggest that the phPS could be considered as a placental embryonic-like stem cell vaccine and that the cutaneous DTH-reaction to phPS is a Th1-cell response to antigens expressed by these cells and which are also cross-reactive with antigens expressed by different epithelial cancer cells. This feature of phPS qualifies it as a multiepitope vaccine-related product that may be used as universal preventive cancer vaccine. Through imparting exogenous and activating endogenous anti-tumor mechanisms within normal healthy individuals by utilizing universal, non-mutated oncofetal antigen vaccines, e.g. phPS vaccine based on embryonic-like stem cells, the immune system is able to destroy nascent cancerous cells before accumulating mutational changes are occuring. The use of overexpressed proteins, as tumor-associated antigens yelds rational targets for specific immunoprevention.

In cancer prophylaxis, we need to destroy just a single cell - the one transformed cell that may give rise to malignancy. Among tumor associated antigens are antigens upregulated in malignant transformation e.g. oncofetal antigens-carcinoembryonic antigen (CEA), alphafetoprotein (AFP), growth factor receptors-Her2/neu, telomerase and p53. A prophylactic vaccination that involves a number of shared antigens may represent a strength of this vaccination approach in as much as immune recognition of multiple antigens would make it less likely for nascent tumor cells to escape immune detection and destruction.

\section{DISCUSSIONS}

\subsection{A Novel Hypoxic Environment Shifts the Antigenic Profile of Adult Stem Cells to One That Is More Embryonic-Like}

A corollary of the stem cell theory of the origin of cancer is that cancers contain the same functional cell populations as normal tissues: stem cells, transit-amplifying cells and mature cells. Cancer tissue differs from normal tissue in that the transit-amplifying cells that do not differentiate to mature cells (maturation arrest) accumulate in cancer, whereas in normal tissue differentiate so that they no longer divide (terminal differentiation) [29]. Unrestained growth and accelerated oxigen consumpton by proliferating stem/progenitor (transit-amplifying) cells of normal tissue, or at distance from blood vessels indicating a hypoxic microenvironment, displays gene expression signatures characteristic of human embryonic stem-like cells, or by prmoting genomic instability drives transformation of stem/progenitor cells also into a stable para-embryonal condition and thus, both may be recognized as dangerous by the immune system. The premise that cancer cells share the expression of oncofetal antigens with stem/progenitor embryonic-like cells and that the immune response against these antigens is cross-protective against cancer and that the disease only manifests if immune response are impeded in protecting against transformation, the use of overexpressed 
proteins of these stem/progenitor embryonic-like cells, as tumor-associated antigens yelds rational targets for specific cancer immunoprevention.

\subsection{The Soluble Forms of Membrane Molecule CD40 (sCD40) May Have a Role in Modulating Antitumor Responses}

Systemic immunity to some gynecological tumors and to syncytiotrophoblast hyperplasia as measured by skin delayed-type hypersensitivity response to phPS strongly support the notion that the host immune system, harboring specific CD40+ $\mathrm{T}$ cells, recognizes the presence of the reexpressed and overexpressed oncofetal antigens (OFAs) on transformed stem/progenitor cells, or on placental stem/progenitor cells reverted to a proliferative embryonic-like stem cell status as result of genomic reprogramming during labor exposure to a novel hypoxic environment, and that can remain alive for days after delivery.

Unfortunately, this proof that the immune system can recognize spontaneous tumors, is for no lasting benefit to the patient. It appears that it is the immune system itself that is hindering its own activity of defense through interference with inhibitory immunological checkpoints controlling $\mathrm{T}$ cell activation.

The ways tumors become unrecognizable to the immune system are various and numerous.

Darrasse-Jèze G., Bergot A-S., et al. [30] emphsize that the relative activation speed of self-specific memory Tregs (regulatorys) versus that of tumor-specific naive Teffs (effector) at the time of tumor emergence dictates tumor outcome. Thus, CD4+-cell responses can also elicit not only stimulatory but also suppressive immunity. It is becoming clear that $\mathrm{T}$ regs play a pivotal role in the tumor progression and the suppression of tumor immunity. Furthermore, effector/memory regulatory $\mathrm{T}$ cells increased as the primary tumor progressed. Data suggest that effector/memory Treg cells are responsible for the loss of concomitant tumor immunity associated with tumor progression [31].

Recently it was shown that $\mathrm{T}$ cell help for CTLs is critically dependent on interaction between CD40L expressed by Th cells and CD40 expressed by APCs.

CD40-CD154 interactions are of central importance and pivotal in the induction of cellular immune responses to many antigens [26]. Several lines of evidence indicate that CD40 signaling is part of an important pathway in $\mathrm{T}$ cell-dependent antigen presentig cell (APC or DC) activation. CD40 ligation on antigen-presenting cells (APCs) by CD40L (CD154) on CD4+ T cells was found to be necessary to induce CD8+ T cell priming by APC. This pathway play a critical role in the induction type- 1 cytokine responses of protective immunity. Dendritic cell
(DC) exist in two stages: Immature and mature. Mature DC cells prime T cells, whereas immature DC can induce tolerance to the presented antigens. The immature DCs are non immunologically quiescent; they have been shown to induce $\mathrm{T}$ cell tolerance in vivo through the induction of $\mathrm{T}$ cell anergy, direct depletion of $\mathrm{T}$ cells, or by generation of regulatory or suppressor cells that block the function of other $\mathrm{T}$ effector $\mathrm{T}$ cells. For full maturation and aquisition of $\mathrm{T}$ cell priming capacity DCs need to be "licensed", which can occur by receiving pro-inflammatory signals in the form of CD4 T cell "help" through CD40-CD40L interaction. Adequate activation of $\mathrm{T}$ cells requires multiple signals from the $\mathrm{DC}$ to the $\mathrm{T}$ cell. MHC-peptide recognised by the $\mathrm{T}$ cell recptor (TCR) on the $\mathrm{T}$ cell is crucial for initial activation, but will lead to anergy or non-responsiveness without appropriate additional costimulation provided by interaction between CD28 on the T cell and B7.1/B7.2 on the DC. Besides help in $\mathrm{CD} 8+\mathrm{T}$ cell priming and maintenance, CD4 $\mathrm{T}$ cells have been shown to recruit and activate various cell populations into the tumor environment, provide bystander mediated killing and affect angiogenesis.

Natural killer (NK) and Natural Killer T (NKT) cells are innate immune cells critical for the first line of defense against tumorigenesis. Although NKT cells possess NK-like cytolytic activity, their activation results in rapid production of IFN- $\gamma$ and expression of CD40L, thus providing help for activation of CD40-expressing APCs and generation of cellular and humoral immune responses.

Therefore, it has been shown that a dominant pathway of CD4+ help is via antigen-presenting cell (APC) activation through engagement of CD40 by CD40 ligand (CD154) on NKT cells and CD4+ T cells. CD40L is mainly expressed transiently on activated CD4+ helper $\mathrm{T}$ cells subsequent to recognition of MHC-peptide complexes. It is required for antigen-presenting cell activetion as it induces costimulatory molecules and cytokine synthesis. It has been suggested that in absence of a strong "danger" signal at this time contributes to the ability of newly forming tumors to avoid recognition by the host immune response. Finally, priming of CD8+ cytotoxic $\mathrm{T}$ lymphocytes generally requires help provided by $\mathrm{CD} 4+$, and the $\mathrm{CD} 40-\mathrm{CD} 40 \mathrm{~L}$ interaction was shown to be essential in the CTL priming via activated DCs.

Also, CD40 is expressed and functional on human epithelial cells, and on these cells, CD40 ligation may be a signal for limitation of cell growth and induction of differentiation [26,27]. Ligation of CD40 on cancer cells was also found to produce a direct growth-inhibitory effect through cell cycle blockage and/or apoptosis with no overt side effects on normal cells. But, the natural antagonist of CD40/CD154 interaction is the soluble form of CD40 (sCD40) which has been shown to inhibit 
the binding of CD154 to CD40 in vitro. High levels of sCD40, which are often over-expressed in tumors, could compete for the ligation of membrane CD40 on CD154, thus resulting in inhibition of antibody production, but also in impeding $\mathrm{T}$ cell activation and in limitation of growth-inhibitory effect of cancer cells by CD 154 on CD40 T cells. The rapid up- and down-regulation of $\mathrm{CD} 154$ on the surface of $\mathrm{T}$ cells is an obvious and important way of control. In a first step, CD154 is quickly expressed upon T-cell receptor engagement and in a second step the CD40 itself contributes to down-regulating CD154 expression on $\mathrm{T}$ cells as sustained interaction between CD40/CD154 leads to endocytosis of the ligand [7]. Given the antagonistic activity of sCD40 on the CD40/CD154 interaction, this shedding mechanism might represent an important negative feedback control of CD40 functions, and thus impairing of CD4 T cells in inducing memory-effector CD8 $\mathrm{T}$ cells (Tc). As all DC types are sensitive to T-cell feedback signals delivered by activated $\mathrm{T}$ cells through CD40 ligand (CD40L) and at least in part by up-regulating CD80/CD86 molecules on APCs, suppression may be generated from fully differrentiated Th1 effector cells by stimulation with antigen in the absence of costimulation.

Barry D. Hock. et al. [32] have shown that CD40 plays a critical role in immunoregulation, suggesting that sCD40 may have a role in modulating antitumor responses and also may be a useful prognostic marker. The release of soluble forms of membrane molecules provides an important mechanism by which cells can either enhance or inhibit the signals delivered by their respective membrane-bound counterparts, suggesting that in vivo release of functional SCD40 would be immunomodulatory. The release of sCD40 by the immune system and/or by malignant cells provides a potentially powerful mechanism for regulating antitumor responses by modulating the interaction of mCD40 with its ligands.

On the other hand effective activation of $\mathrm{T}$ cells requires engagement of two separate T-cell receptors. The antigen-specific T-cell receptor (TCR) binds foreign peptide antigen-MHC complexes, and the $\mathrm{CD} 28$ receptor binds to the $\mathrm{B} 7$ (CD80/CD86) costimulatory molecules expressed on the surface of antigen-presenting cells (APC). The simultaneous triggering of these T-cell surface receptors with their specific ligands results in an activation of this cell. In contrast, CTLA-4 (CD152) is a distinct T-cell receptor that, upon binding to B7 molecules, sends an inhibitory signal to $\mathrm{T}$ cell activation. CD86 could be more important for initiating T-cell responses, while CD80 could be more significant for maintaining these immune responses [33].

This interpretation assumes that CD28 functions not as an "on-off" switch but rather as a kind of "rheostat" which, depending on the strength and/or duration of its engagement by B7 molecules, can display a degree of plasticity in the intracellular signals it generates [33].

Most $\mathrm{CD} 4{ }^{+} \mathrm{T}$ cells belong to either the Th1 or Th2 subsets. However $\sim 10 \%$ of them do not. These so-called T-regulatory (Treg) cells. The CTLA- 4 molecules on Treg cells bind very tightly to the $\mathrm{B} 7$ molecules on antigen-presenting dendritic cells and B cells. Once bound, they kill the target (by secreting perforins). Treg cells can also kill cytotoxic T lymphocytes (CTL) and natural killer (NK) cells.

Consistent with a B7 "competition model" because CTLA-4 has been estimated to have 50 - 100-fold higher affinity to B7 than $\mathrm{CD} 28$, it is conceivable that the T reg cells, are competitively engaging available $\mathrm{B} 7$ molecules on the APCs, thereby preventing CD28 signaling, and subsequent $\mathrm{T}$ cell activation in effector $\mathrm{T}$ cells. [34] The co-existence of tumor specific immunity with a progressing tumor is observed in a variety of experimental systems and remains one of the major paradoxes of tumor immunology. It appears that it is the immune system itself that is hindering its own activity of defense. This behavior marks a resemblance to that of the embryo and of cancer [35].

The above observations lead to the conclusion that reexpression and overexpression of the trophoblast crossreactive antigens present on placental hyperplasic cells, but also on neoplastic cells and continuously shedding of CD40 (sCD40) represent an adaptive response of these cells to natural selection pressures as a biological response to resist immunological recognition and rejection by the host. Inhibition of the CD/E (expressing) 40 pathway by increased levels of soluble CD40 receptor (sCD40) which reduces availability, may impede the immune system from mounting appropriate humoral and cell-mediated responses against precancerous cells Given the antagonistic activity of sCD40 on the CD40/CD154 interaction, this shedding mechanism might represent an important negative feedback control of CD40 functions, suggesting that $\mathrm{SCD} 40$ may have a role in modulating a pre-existing or de novo anti-tumor responses through interference with inhibitory immunological check points controlling $\mathrm{T}$ cell activation.

\subsection{Placental Stem/Progenitor Embryonic-Like Cells Based Cellular Vaccine an Efficient Immuno Preventive of Human Malignancies}

To overcome these escape mechanisms and to acquire the goal of an optimal adaptive immune response particularly to reexpression of embryonic proteins that begin to be overexpressed in tumor cells early in their transformation and which can be considered as non-self by the immune system, it proposed a vaccination strategy design by intradermal injection of a pharmaceutical hu- 
man allogeneic Placenta Suspension (phPS), prepared upon Filatov's method and admixed with BCG Vaccine ("danger" signal) in normal healthy individuals to induce activated/memory $\mathrm{T}$ effector cells (amTeffs). This crosspriming of the tumor cells overexpressed antigen-specific response by potent APC is a major mechanism of the developing integrated endogenous immune response, thereby shifting the balance from tolerance to activation induction, and/or rejuvenating functionally inferior responses of the exausted T cells.

Activated Th1 cells have longevity compared with other activated immune cells (APCs and CTL) and are conventionally viewed as responsible for immune memory, capable of revitalizing the immune response if a specific antigen is reencountered, including promtly function of CD40/CD40L interaction, prior to the release of sCD40 by the immune system and/or by transformed cells. The presence of activated/memory $\mathrm{T}$ effector cells (amTeffs) at the time of tumor emergence shifts the Treg/ Teff balance toward efficient antitumor immune response. Alternatively, or in addition, the CD4 T cells may be required for activation of macrophages and dendritic cells, an event crucial for killing of transformed cells. This antisupressive approach reverses the host's immune factors that confer a survival advantage to tumor emergence and require an intact immune system to function.

The biological adjuvant Bacillus Calmette GuerinVaccine (BCG-Vaccine) takes advantage of the fact that it is an immunogenic compound, naturally recognized as foreign and known to induce migration of APCs to the site of delivery. APCs responding to the adjuvant stimulation are thus able to coincidentally capture and process placental antigens present in the inflammatory milieu.

Becker Th. et al. [36] have suggested that CD40 is a cochaperone-like receptor mediating the uptake of exogenous Hsp70-peptide complexes by macrophages and dendritic cells. For the generation of potent $\mathrm{T}$ cell immune responses against self-tumor Ags, Hsp70 Ag from Mycobacterium tuberculosis was shown to induce Th1polarized cytokine responses and activate DCs via binding with CD40 (perhaps via promoting cross-talk between various inflammatory cells recruited at the vaccine site). Although the Ag targeting alone, without activation of dendritic cells (DCs), is proposed to induce tolerance, the vaccines expressing self-tumor Ags is rendered immunogenic if targeted to the APCs using mycobacterial Hsp70 Ag [36] and thus, eliciting long-term protective $\mathrm{CD} 8+\mathrm{T}$ cell-mediated memory responses effective in killing the emerging tumor cells.

At the same time the immune response is mediated through the release of different cytokines which can influence the synthesis and actions of one another in the setting of an immunoregulating cytokine network, shaping an environment in which the presence of active/ memory $\mathrm{T}$ effector cells (am Teffs) at the very time of tumor emergence, are able to bypass the tumor immunity mediated by self-specific memory regulatory $\mathrm{T}$ cells. These two aspects of a preventive vaccination with multiepitope phPS/BCG-Vaccine might be useful for generating an immune response against a broad-spectrum of cancers, thus serving as a universal cancer vaccine.

The possibility of inducing long-term protection against tumors by vaccination at the earliest signs of its development has the potential to cause a dramatic paradigm shift in the prevention of tumors.

The rationale for prevention is strong because, in that setting one deals with an immune system that is neither impaired by tumor- and treatment-induced suppression nor tolerant to tumor-associated antigens that have been encountered in the absence of correct presentation and costimulatory/danger signals. On the other hand, a future success of cancer prevention will depend on how effectively a preventive vaccination strategy simultaneously acts on emerging pretumor cells as well as on its microenvironment [37].

Conceptually, to avoid the regulatory T cells' immune suppression that develops in tumor-bearing patients, vaccination with a pharmaceutical allogenic human Placenta Suspension prepared upon Filatov's method, after a "danger signal" is added e.g. BCG-Vaccine, would be capable of eliciting immunological responses in normal healthy individuals, not only to marker antigens shared between the Placenta-Suspension preparation and neoplasia, but also by introducing a new environment of Tcell-induced cytokines, that provide a more "complete" immune response, to prevent tumor development far into future, by inhibition, or by elimination of transformed cells at their earliest manifestation. Thus, active immunoprevention uses the host's immune cells and requires an intact immune system to function.

Using of isolated labor intermittent hypoxia-induced placental stem/progenitor embryonic-like cells of afterbirth placenta per se, or of phPS prepared upon Filatov's method, as a vaccine-related product for a preventive cancer vaccine, warrants further investigation.

The above hypothesis is rising as a challenge to the scientific community in the hope that it will cause an examination of this matter.

\section{REFERENCES}

[1] Corocleanu, M. (1981) Humoral and cellular reactions to placental antigens in women with pathological pregnancies and cancer patients. Revue Roumaine de Biochimie, 18, 7-13.

[2] Corocleanu, M. (2008) A possible "universal" cancer vaccine that might cause an immune response against emerging cancer cells that originate from any tissue. Medical Hypothesis, 70, 381-383. 
http://dx.doi.org/10.1016/j.mehy.2007.04.040

[3] Redman, C.W.G. (1986) Immunology of the placenta. Clinical Obstetrics and Gynecology, 16, 469.

[4] Hamilton, M.S. (1983) Maternal immune responses to oncofetal antigens. Journal of Reproductive Immunology, 5, 249. http://dx.doi.org/10.1016/0165-0378(83)90252-8

[5] Sarandakou, A., et al. (2007) Tumor markers in biological fluids associated with pregnancy. Critical Reviews in Clinical Laboratory Sciences, 44, 151-178. http://dx.doi.org/10.1080/10408360601003143

[6] Marilyn, M., et al. (2001) Human embrionic genes reexpressed in cancer cells. Oncogene, 20, 8085-8091. http://dx.doi.org/10.1038/sj.onc.1205088

[7] Yu, J.Y., et al. (2007) Induced pluripotent stem cell lines derived from human somatic cells. Science, 318, 19171920. http://dx.doi.org/10.1126/science. 1151526

[8] Coggin Jr., J.H., Barsoum, A.L., Rohrer, J.W., Tucker, J.A. and Dyess, D.L. (2001) Materno-fetal immunobiology hypothesis: Immature laminin receptor protein is a primitive, species conserved, universal embryonic $\mathrm{T}$ and $\mathrm{B}$ cell immunogenic providing both maternal and fetal protection. Modern Aspects of Immunobiology, 2, 84-91.

[9] Coggin Jr., J.H. and Anderson, N.G. (1974) Cancer, differentiation and embryonic antigens: Some central problems. Advances in Cancer Research, 19, 105-165. http://dx.doi.org/10.1016/S0065-230X(08)60053-6

[10] Siegel, S., et al. (2006) Identification of HLA-A*0201presented $\mathrm{T}$ cell epitopes derived from the oncofetal antigen-immature laminin receptor protein in patients with hematological malignancies. The Journal of Immunology, 176, 6935-6944.

[11] Rohrer, J.W., et al. (1999) Human breast carcinoma patients develop clonable oncofetal antigen-specific effector and regulatory $\mathrm{T}$ lymphocytes. Journal of Immunology, 162, 6880-6892.

[12] Lee, M.W., et al. (2010) Stem and progenitor cells in human umbilical cord blood. International Journal of Hematology, 92, 45-51.

http://dx.doi.org/10.1007/s12185-010-0619-4

[13] Zhong, Z., et al. (2009) Feasibility investigation of allogeneic endometrial regenerative cells. Journal of Translational Medicine, 7, 15. http://dx.doi.org/10.1186/1479-5876-7-15

[14] Adelman, D.M., et al. (2000) Placental cell fates are regulated in vivo by HIF-mediated hypoxia responses. Genes \& Development, 14, 3191-3203.

[15] Fryer, B.H., et al. (2006) Hypoxia, HIF and the placenta, Cell Cycle, 5, 495-498. http://dx.doi.org/10.4161/cc.5.5.2497

[16] Pugh, C.W., et al. (2003) Regulation of angiogenesis by hypoxia, role of the HIF system. Nature Medicine, 9, 677-684. http://dx.doi.org/10.1038/nm0603-677

[17] Myatt, L., et al. (2004) Oxidative stress in the placenta, Histochemistry and Cell Biology, 122, 369-382. http://dx.doi.org/10.1007/s00418-004-0677-x

[18] Vaiman D. et al. (2005) Hypoxia-activated genes from early placenta are elevated in pre-eclampsia, but not in intra-uterine growth retardation. BMC Genomics, 6, 111. http://dx.doi.org/10.1186/1471-2164-6-111

[19] Shigeru, S., et al. (2003) Th1/Th2 balance in preeclampsia. Journal of Reproductive Immunology, 59, 161-173. http://dx.doi.org/10.1016/S0165-0378(03)00045-7

[20] Darmochwal-Kolarz, D., et al. (2007) Activated T lymphocytes in pre-eclampsia. American Journal of Reproductive Immunology, 58, 39-45. http://dx.doi.org/10.1111/j.1600-0897.2007.00489.x

[21] Sasaki, Y., et al. (2007) Proportion of peripheral blood and decidual $\mathrm{CD}(4+) \mathrm{CD} 25$ (bright) regulatory $\mathrm{T}$ cells in pre-eclampsia. Clinical \& Experimental Immunology, 149, $139-145$ http://dx.doi.org/10.1111/j.1365-2249.2007.03397.x

[22] Cindrova-Davies, T., et al. (2007) Oxidative stress, gene expression, and protein changes induced in the human placenta during labor. American Journal of Pathology, 171, 1168-1179. http://dx.doi.org/10.2353/ajpath.2007.070528

[23] Schmitt, E., et al. (2007) Intracellular and extracellular functions of heat shock proteins: Repercussion in cancer therapy. Journal of Leukocyte Biology, 81, 15-27. http://dx.doi.org/10.1189/jlb.0306167

[24] Srivastava, P. (2002) Interaction of heat shock proteins with peptides and antigen-presenting cells: Chaperoning of the innate and adaptive immune responses. Annual Review of Immunology, 20, 395-425.

http://dx.doi.org/10.1146/annurev.immunol.20.100301.06 $\underline{4801}$

[25] Yoshino, I., et al. (1994) Human tumor-infiltrating CD4+ $\mathrm{T}$ cells react to $\mathrm{B}$ cell lines expressing heat sock protein 70. The Journal of Immunology, 153, 4149-4158.

[26] Young, L.S., et al. (1998) CD40 and epithelial cells: Across the great divide. Immunology Today, 19, 502. http://dx.doi.org/10.1016/S0167-5699(98)01340-1

[27] Peguet-Navarro, J., et al. (1997) CD40 ligation of human keratinocytes inhibits their proliferation and induces their differentiation. The Journal of Immunology, 158, 144152.

[28] Contin Cécile, J., et al. (2003) Membrane-anchored CD40 is processed by the tumor necrosis factor- $\alpha$-converting enzyme. Journal of Biological Chemistry, 278, 3280132809. http://dx.doi.org/10.1074/jbc.M209993200

[29] Stewart, S. (2008) Alpa-fetoprotein, stem cells and cancer: How study of the production of alpha-fetoprotein during chemical hepatocarcinogenesis led to raffirmation of the stem cell theory of cancer. Tumor Biology, 29, 161-180. http://dx.doi.org/10.1159/000143402

[30] Darrasse-Jèze, G., Bergot, A.-S., et al. (2009) Tumor emergence is sensed by self-specific CD44hi memory Tregs that create a dominant tolerogenic environment for tumors in mice. Journal of Clinical Investigation, 119, 2648-2662.

[31] Goreliki, E. (1983) Concomitant immunity and the resistance to a second tumor challenge. Advances in Cancer Research, 39, 71-120.

http://dx.doi.org/10.1016/S0065-230X(08)61033-7

[32] Hock, B.D., et al. (2006) Circulating levels and clinical 
significance of soluble CD40 in patients with hematological malignancies. Oncology \& Radiotherapy, 106, 2148-2157.

[33] Prilliman K.R., et al. (2002) Cutting edge: A crucial role for B7-CD28 in transmitting T help from APC to CTL. The Journal of Immunology, 169, 4094-4097.

[34] Peggs Karl, S., et al. (2009) Blockade of CTLA-4 on both effector and regulatory $\mathrm{T}$ cell compartments contributes to the antitumor activity of anti-CTLA-4 antibodies. Journal of Experimental Medicine, 206, 1717-1725. http://dx.doi.org/10.1084/jem.20082492

[35] Vasilevko, V., Ghochikyan, A., Holterman, M.J. and
Agadjanyan, M.G. (2002) CD80 (B7-1) and CD86 (B7-2) are functionally equivalent in the initiation and maintenance of $\mathrm{CD} 4+\mathrm{T}$-cell proliferation after activation with suboptimal doses of PHA. DNA and Cell Biology, 21, 137-149. http://dx.doi.org/10.1089/10445490252925404

[36] Becker, Th., et al. (2002) CD40, an extracellular receptor for binding and uptake of Hsp70-peptide complexes. The Journal of Cell Biology, 158, 1277-1285. http://dx.doi.org/10.1083/jcb.200208083

[37] Finn, O.J., et al. (2002) Prophylactic cancer vaccines. Current Opinion in Immunology, 14, 172-177. http://dx.doi.org/10.1016/S0952-7915(02)00317-5 